\title{
Cardioneuroablation using an anatomical approach: a new and promising method for the treatment of cardioinhibitory neurocardiogenic syncope
}

\author{
Roman Piotrowski, Jakub Baran, Piotr Kułakowski \\ Postgraduate Medical School, Department of Cardiology, Grochowski Hospital, Warsaw, Poland
}

\section{INTRODUCTION}

Neurally mediated syncope (NMS) is the most common cause of syncope and is brought about by enhanced vagal tone leading to asystole, sinus bradycardia, or atrioventricular block (AVB) [1]. According to the current guidelines, dual-chamber cardiac pacing may be considered in patients $>40$ years old, when the correlation between syncopal episodes and asystole is documented. In younger patients, pacemaker implantation may be considered only in very selected cases [1].

Recently, endocardial biatrial ablation of ganglionated plexi (GP) has been shown to provide excellent short- and long-term results in the treatment of syncope caused by cardioinhibitory reflex syncope or functional AVB [2-4]. However, this method, called cardioneuroablation (CNA), is still evolving, and more data are needed to establish its role in the treatment of NMS.

We present three patients with frequent syncopal episodes due to cardioinhibitory mechanism, who underwent CNA in order to improve symptoms and avoid pacemaker implantation.

\section{METHODS \\ Mapping and ablation}

Mapping and ablation were performed under mild sedation (midazolam and fentanyl), using a 3.5-mm irrigated-tip catheter (Navistar ThermoCool SmartTouch; Biosense Webster, Diamond Bar, CA, USA) with contact force module and the electroanatomical system Carto 3 (Biosense Webster). Electroanatomical maps of the right atrium (RA) and left atrium (LA) were created, and anatomical ablation of autonomic ganglia was performed. In all cases ablation in the RA was started from the superoposterior area (superior right atrial GP), followed by the mid-posterior area (posterior right atrial GP) and the inferoposterior area (inferior right GP). In the LA, ablation was started at the site of the anterior right GP and was continued downwards along the anterior part of a common vestibulum of the right pulmonary veins (PVs), opposite the right-sided ablation lesions. We did not ablate regions corresponding to the left PV. We also did not perform high-frequency stimulation (HFS) to identify the location of the GP. In three initial cases described herein we did not use atropine test; however, currently we use it in all patients. This test can identify proper candidates for the procedure because atropine suppresses the parasympathetic system and predicts the effects of CNA.

Our approach was purely anatomical, with attention paid to ablation-induced responses such as asystole and/or an increase in sinus rate. All patients gave written, informed consent to undergo CNA.

\section{Case 1}

A 26-year-old man without structural heart disease and with a negative initial neurological work-up was referred for evaluation of recurrent syncopal episodes. In-hospital telemetry revealed a 16-s asystole associated with syncope. The symptoms were reproduced during tilt test (TT), i.e. a 16-s asystole causing syncope was induced (Fig. 1). Despite following general recommendations such as lifestyle measures, increase of water intake, and isometric manoeuvres, the patient continued to suffer from recurrent syncopal events. He was referred to our centre for pacemaker implantation. After a discussion with the patient and his family, we decided to perform CNA. During ablation sinus rhythm (SR) increased from 80 to $120 \mathrm{bpm}$. After the procedure, a control TT was performed and no cardioinhibitory response was observed. Four months after CNA syncopal episodes recurred, and so a dual-chamber pacemaker (CLS, Biotronik, Berlin, Germany) was implanted. Despite this, episodes of transient loss of consciousness (TLOC) still recurred. Moreover, the device did not

\section{Address for correspondence:}

Roman Piotrowski, MD, Postgraduate Medical School, Department of Cardiology, Grochowski Hospital, ul. Grenadierow 51/59, 04-073 Warszawa, Poland, tel: +48 225152 757, e-mail: rpiotrow@op.pl

Received: 9.08.2018 Accepted: 24.09.2018

Available as AoP: 25.09.2018

Kardiologia Polska Copyright (c) Polish Cardiac Society 2018 


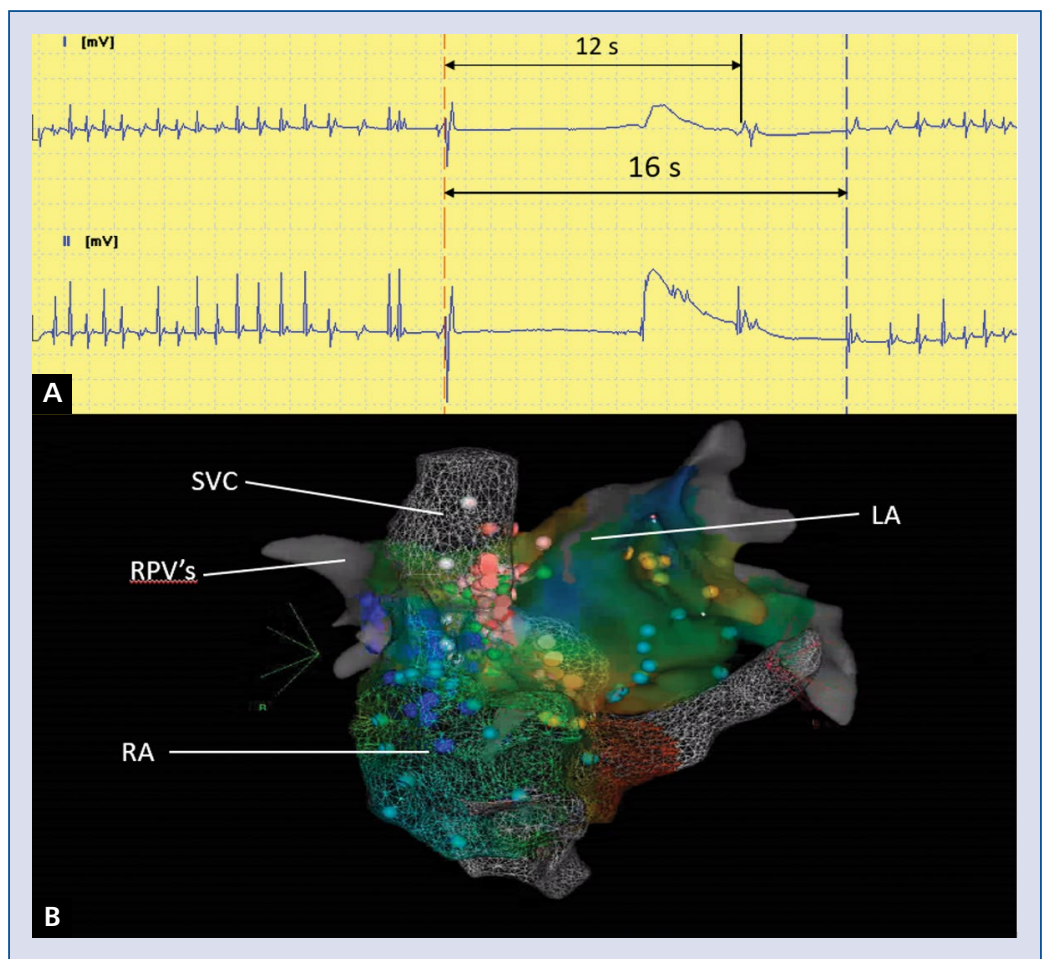

Figure 1. Electrocardiogram with asystole induced during a tilt test (A) and an electroanatomical map during cardioneuroablation (B). Red dots show applications in the right atrium (RA), green dots show applications in the left atrium (LA), and blue dots show the course of the phrenic nerve. SVC — superior vena cava, RPVs — right pulmonary veins

record any periods of atrial or ventricular pacing, which implied that the mechanisms of loss of consciousness might have been other than vasovagal. Repeated detailed neurological examination suggested epilepsy and treatment with valproic acid was started. Since then, no TLOC episodes were noted, and after 12 months without TLOC the pacemaker was explanted. During the 10 months after pacemaker explantation no syncopal episodes/presyncope were observed.

This case shows two independent causes of TLOC. Effective CNA eliminated enhanced vagal tone and NMS, whereas antiepileptic medication effectively prevented recurrence of seizures. It also shows that in difficult cases repeated investigations such as neurological evaluation, as demonstrated herein, may be of value and may change the initial diagnosis. The decision to explant the pacing system, which is often difficult in clinical practice, was based on the assumption that both causes of TLOC, vasovagal reaction and epilepsy - were effectively treated.

\section{Case 2}

A 43-year-old man with epilepsy and without structural heart disease was referred to our centre due to recurrent syncopal episodes and documented 42-s asystole during TT.

After obtaining written, informed consent we performed $\mathrm{CNA}$. Asystole during application in the mid-posterior RA area was observed. During application at the site of the anterior right GP of LA, an increase in SR from 60 to $75 \mathrm{bpm}$ was observed. Control TT after CNA revealed no cardioinhibitory response. No syncopal episodes were noted in a 10-month follow-up.

\section{Case 3}

A 44-year-old man without structural heart disease was referred to our centre for pacemaker implantation due to recurrent syncope. Baseline Holter electrocardiogram (ECG) monitoring showed permanent first-degree AVB and paroxysmal second-degree Wenckebach-type AVB (over 600 episodes of second-degree AVB and 29 pauses over 2 s). During TT cardioinhibitory response with 8-s asystole and syncope were noted. During ablation, the degree of AVB decreased from second to first with PR interval of $213 \mathrm{~ms}$, and an increase in SR from 70 to $95 \mathrm{bpm}$ was noted. Holter ECG after CNA showed only first-degree AVB with a shorter PR interval of $213 \mathrm{~ms}$. SDNN decreased from 122 to $99 \mathrm{~ms}$ whereas mean heart rhythm remained unchanged during Holter ECG after CNA. Thus, CNA affected the lower ganglia, responsible for atrioventricular conduction, to a greater extent than the upper ganglia, responsible for SR. No electrophysiological study was performed. During seven months of follow-up no syncopal episodes and no AVB higher than first degree were observed. 


\section{DISCUSSION}

Recently, CNA has been suggested as an effective and safe method for the treatment of vasovagal syndrome (VVS) caused by cardioinhibitory or mixed mechanism [2-4]. Pachon et al. [2] reported excellent long-term outcomes in 43 patients. Similar findings were reported by Sun et al. [3] in 57 patients. In another study, Aksu et al. [4] showed that CNA was effective in patients with VVS, caused by second- and third-degree AVB. In addition, a few case reports also showed efficacy and safety of CNA [5, 6]. The 2018 European Society of Cardiology guidelines [1] are the first official document in which CNA was mentioned as the potential method for treatment of VVS; however, it has not received any recommendation class.

The endpoints were different in all cited studies. Pachon et al. [2] defined endpoints of CNA as elimination of the potentials with right spectral shift in the RA and LA regions surrounding the sinus and atrioventricular nodes, persistent increase in SR and the Wenckebach point, and anatomical ablation. Aksu et al. [4] assumed that elimination $(<0.1 \mathrm{mV})$ of the atrial fractionated potentials above $300 \mathrm{~Hz}$, elimination of parasympathetic response to HFS, persistent increase in SR and the Wenckebach point, and complete elimination of AVB were endpoints during CNA [4]. Elimination of all vagal responses at each identified target was assumed as an endpoint used by Sun et al. [3].

The optimal technique for performing CNA has not been established yet. For identification of GP sites, spectral mapping, HFS, or anatomical approach have been used [2-4]. The HFS affects ganglia more strongly than standard pacing and helps to identify GPs with greater precision. However, anatomically guided ablation has been shown to produce similar results [3]. More detailed methods for localising GP are needed. There are initial reports suggesting that specific markers such as ${ }^{123} \mathrm{I}$-mIBG (metaiodobenzylguanidine) guided by Spectrum Dynamics (D-SPECT) may identify the anatomical site of GP in a given patient [7]. Also, the optimal procedural endpoint has not been well established. There is no universally accepted target of SR acceleration following
CNA. A 20\% increase in SR is often used as a cut-off because such an increase suggests that appropriate suppression of parasympathetic activity was achieved.

In our patients the procedure was stopped when an increase in heart rhythm was obtained or the degree of AVB was reduced. Left-sided regions with GP near the left PV were not ablated. Ablation near left PVs is only considered if ablation at the RA and right PVs is ineffective. The procedure should be minimally invasive.

\section{Conflict of interest: none declared}

\section{References}

1. Brignole M, Moya A, de Lange FJ, et al. 2018 ESC Guidelines for the diagnosis and management of syncope. Eur Heart J. 2018; 39(21): 1883-1948, doi: 10.1093/eurheartj/ehy071, indexed in Pubmed: 29562291.

2. Pachon JC, Pachon EI, Cunha Pachon MZ, et al. Catheter ablation of severe neurally meditated reflex (neurocardiogenic or vasovagal) syncope: cardioneuroablation long-term results. Europace. 2011; 13(9): 1231-1242, doi: 10.1093/europace/eur163, indexed in Pubmed: 21712276.

3. Sun W, Zheng L, Qiao Yu, et al. Catheter ablation as a treatment for vasovagal syncope: long-term outcome of endocardial autonomic modification of the left atrium. J Am Heart Assoc. 2016; 5(7), doi: 10.1161/JAHA.116.003471, indexed in Pubmed: 27402231.

4. Aksu T, Golcuk E, Yalin K, et al. Simplified cardioneuroablation in the treatment of reflex syncope, functional AV block, and sinus node dysfunction. Pacing Clin Electrophysiol. 2016; 39(1): 42-53, doi: 10.1111/pace.12756, indexed in Pubmed: 26411271.

5. Fukunaga M, Wichterle D, Peichl P, et al. Differential effect of ganglionic plexi ablation in a patient with neurally mediated syncope and intermittent atrioventricular block. Europace. 2017; 19(1): 119-126, doi: 10.1093/europace/euw100, indexed in Pubmed: 27194540.

6. Rebecchi M, de Ruvo E, Strano S, et al. Ganglionated plexi ablation in right atrium to treat cardioinhibitory neurocardiogenic syncope. J Interv Card Electrophysiol. 2012; 34(3): 231-235, doi: 10.1007/s10840-012-9666-5, indexed in Pubmed: 22434334.

7. Romanov A, Minin S, Breault C, et al. Visualization and ablation of the autonomic nervous system corresponding to ganglionated plexi guided by D-SPECT I-mIBG imaging in patient with paroxysmal atrial fibrillation. Clin Res Cardiol. 2017; 106(1): 76-78, doi: 10.1007/s00392-016-1045-2, indexed in Pubmed: 27752762.

Cite this article as: Piotrowski R, Baran J, Kułakowski P. Cardioneuroablation using an anatomical approach: a new and promising method for the treatment of cardioinhibitory neurocardiogenic syncope. Kardiol Pol. 2018; 76(12): 1736-1738, doi: 10.5603/KP.a2018.0200. 